\title{
Genomic Instability or One-Gene Theory for Tumor Progression: A Breast Cancer Study
}

\section{Roland B Sennerstam ${ }^{1 *}$ and Jan-Olov Strömberg ${ }^{2}$}

${ }^{1}$ Department of Pathology and Oncology Karolinska Hospital and Karolinska Institutet SE-171 76 Stockholm, Sweden

${ }^{2}$ Department of Mathematics, Royal Institute of Technology, SE-100 74 Stockholm, Sweden

\begin{abstract}
Objective: There is an ongoing debate in the literature as to whether human cancers originate from unique clones with single oncogene mutations or propagate from early established genomic instabilities due to intermediate metastable tetraploidization. The aim of this study was to investigate how far genomic instability, reflected in ploidy alterations, can explain tumor progression.

Methods: In total 1,280 patients were involved in this study. We defined DNA-index (DI) intervals for diploid, tetraploid and aneuploid tumors and made simulations based on increasing age of patients, from 30 to 60 years old. We related this information to four enhancement steps of a parameter reflecting genomic instability generated from the tumor G1 peak coefficient of variation, S-phase fraction and number of cells exceeding G2 phase DNA region (stemline-scatter-index; SSI). The change in ploidy entities was also simulated with respect to growing values of the parameter for genomic instability (SSI).

Results: Following the age-dependent alteration in ploidy there were, at the lowest level of genomic instability up to 45 years of age, only diploid (87\%) and tetraploid (13\%) tumors. In three SSI relative unit enlargements, along with increasing age, aneuploid tumors were mainly found to be derived from tetraploid tumors resulting in a growing number of hypotetra and hypertriploid tumors. The hypertriploid tumors $(1.4 \leq \mathrm{DI}<1.8)$ increased 23 -fold during the age interval 35 to 60 years, and a strong correlation was found between genomic instability and hypertriploid tumors. In the simulation experiments, it was found that tetraploidization occurred twice during tumor progression and it generated two populations of aneuploid tumors.
\end{abstract}

Conclusion: Our analysis indicates that genomic instability originates mainly in tetraploid tumors, in which a state of high genomic instability results in the loss of genetic material due to mitotic failure. This generates selective competence and enhances the aggressiveness of tumors.

Keywords: Tumor progression; Breast cancer; Genomic instability; DNA-ploidy; Tetraploidization; Aneuploidy; Tumor hypoxia

Abbreviations: BC: Breast Cancer; DI: DNA-Index; D-Type Tumors: Diploid Tumors; T-Type Tumors: Tetraploid Tumors; A-Type Tumors: Aneuploid Tumors; A1-Type Tumors: A-Type Tumors With DNA-Index $1.2 \leq \mathrm{DI}<1.4$; A2-Type Tumors: Aneuploid Tumors With DNA-Index $1.4 \leq \mathrm{DI}<1.8$; A3-Type Tumors: Aneuploid Tumors With DNA-Index $\geq 2,2$; SSI: Stemline-Scatter-Index; G1-Phase: Gap1 Phase Before SPF; G1CV: Coefficient Of Variation Of G1 Peak; SPF: S-Phase Fraction; G2-Phase: Gap 2 After SPF; Ex-G2: Cells Of DNAValues Above DI $\geq 2$.2: PI: Proliferation Index: SPF+G2-Phase; CGH: Comparative Genomic Hybridization.

\section{Introduction}

Polyploidization is an evolutionarily conserved phenomenon that may confer survival advantages over diploidy by facilitating adaptation to various environmental and nutritional stresses $[1,2]$. DNA analysis has determined that it first evolved on the earliest branches of the phylogenetic tree [3]. Polykaryocytosis was initially identified in 1965 following infection of human cell lines with the measles virus [4]. Tetraploidization has since been detected in humans in response to various stresses, such as an overloaded left heart, congenital heart disease and hypertension [5,6], pregnancy (as detected in the uterus) [7], hepatocyte regeneration after injury, metabolic overload and oxidative stress [8], and inflammatory bronchi [9]. Tetraploidization of the vascular smooth muscle [10], aged endothelial and fibroblast cells has also been identified $[11,12]$. However, the whole genome in mammals is generally diploid. There are, however, rare exceptions, for example the red vizcacha rat (Tympanoctomys barrerae) and close relatives to this rat are tetraploid [13]. In plants, amphibians, fish and yeast, whole genome tetraploidy is common [2]

During carcinogenesis, tetraploidy is regarded as an intermediate metastable state between euploid and aneuploid tumors [14,15-19]. In tetraploid cells, the supernumerary centrosomes create multipolar spindle formation and increase the risk of disrupted chromosome segregation and mitotic failure that will destabilize the karyotype and generate hyper-, hypotetraploid and hypertriploid cells [20-22]. Tetraploidy has been proposed to be tumorigenic in its own right [23]. In mild colon adenomas, $27 \%$ of aneuploid cells were found without p53 immunoreactivity and only 50\% had a diploid DNA histogram. In moderate colon adenomas $43 \%$ were aneuploid, $17 \%$ stained positive for p53 and 39\% were diploid [24]. These data show that p53 mutation can occur after initiation of genomic destabilization, raising the question as to whether genomic instability is the main driving force in tumor

*Corresponding author: Roland B Sennerstam, Department of Pathology and Oncology Karolinska Hospital and Karolinska Institutet SE-171 76 Stockholm, Sweden Tel: 0046-8-745 54 08; Fax: 0046-8-331-696; E- mail: roland.sennerstam@ki.se

Received February 14, 2015; Accepted April 21, 2014; Published April 27, 2015

Citation: Sennerstam RB, Strömberg JO (2015) Genomic Instability or One-Gene Theory for Tumor Progression: A Breast Cancer Study. J Carcinogene Mutagene 6: 223. doi:10.4172/2157-2518.1000223

Copyright: (c) 2015 Sennerstam RB, et al. This is an open-access article distributed under the terms of the Creative Commons Attribution License, which permits unrestricted use, distribution, and reproduction in any medium, provided the original author and source are credited. 
progression [25-27], rather than the prevailing gene-based cancer theory [28] and does aneuploidy cause cancer? [29,30]. Chromosomal instability (CIN) has been established in the literature in contrast to the genecentric concept [31] dominating cancer research since the identification of the Philadelphia chromosome involved in chronic myelocytic leukemia (CML). Analyzing comparative genomic hybridization (CGH) gains, losses and segmental amplifications of chromosomes appeared in higher frequency and with more chromosomes involved in tumors with increased malignancy potential and shorter survival [32]. CGH changes occur at a rate that far exceeds those at which genotypes are changed by conventional mutations [33,34].

In tumors that are established and of a certain size, hypoxia has been proposed to be a selection factor driving further increases in genomic instability $[35,36]$; i.e., the already established genomic heterogeneity generates many new subclones that can survive hypoxia-related stress factors in the prevailing environment. Solid tumors contain microenvironments of low nutrient availability, and can suffer from low extracellular $\mathrm{pH}$, and hypoxia [37]. This is particularly true before tumor vascularization has reached a level sufficient to supply adequate nutrition and oxygen to the continuously growing whole tumor mass. Consistently, over-expression of hypoxia-inducible factor-1a (HIF-1 $\alpha$ ) has been shown to be a predictive marker of early relapse in breast cancer [38]. Furthermore hypoxia affects growth, metastatic potential, and the response to therapy in breast cancer [39]. Additionally, the level of vascular endothelial growth factor (VEGF) was reported to be low in small breast tumors $(<20 \mathrm{~mm})$ but much higher in larger tumors [40].

A large number of studies investigating tumor DNA ploidy were reported in the last three decades of the 20th century [41,42-45]. Reports of this type have declined from around the year 2000, in favor of studies using micro-molecular markers and cancer specific genes. However, DNA-ploidy data can be used to study the issue of genomic instability and its impact on tumor progression. In this report we used data from Feulgen-stained tumor cells to make simulations of the interactions and the sequences of appearance of diploid (D-type), tetraploid (T-type) and aneuploid (A-type) tumors. The aim of the study was to determine whether genomic instability might by itself be a driving force towards higher levels in tumor heterogeneity. Genetic instability of cancer cells has been found to be proportional to their degree of aneuploidy [46] and in this report we continue to focus on the broad status of ploidy change in breast cancer patients during tumor progression.

\section{Materials and Methods}

\section{Study population}

We analyzed a randomized sample of 1,280 breast cancer (BC) patients diagnosed from 1991 to 1993 from whom follow-up data for up to 18 years were available. DNA data and proliferation indices (S-, and G2-phases) were obtained from all patients. Tumor size was available from $98 \%$ of the study population. Women aged 50-69 years were invited for mammography screening every second year, and when women were randomly interviewed in the early nineties in total 85$90 \%$ had underwent one mammography investigation within the last two years. Since the study was focused on the dynamics of DNA-ploidy change during tumor progression tumor grade was not included in the study. The entire population was subdivided into DI entities based on DI intervals: (1) Diploid (D-type) $(0.9 \leq \mathrm{DI}<1.2, \mathrm{n}=607)$; (2) Aneuploid (A-type) $(1.2 \leq \mathrm{DI}<1.8,(\mathrm{n}=258)$; and (3) Tetraploid (T-type) tumors $(1.8 \leq \mathrm{DI}<2.2, \mathrm{n}=342)$. For some parts of the analysis, the A-type tumors were divided into A1-type $(1.2 \leq \mathrm{DI}<1.4)$, A2-type $(1.4 \leq \mathrm{DI}<1.8)$ and a small A3-type group with DI $\geq 2.2(n=68)$. Our classification of $D$ - and
T-type tumors covers DI ranges slightly larger than those proposed in other publications [47] because we found it to better fit the analysis of increasing genomic instability. The A3-type group with $\mathrm{DI} \geq 2.2$ was excluded from some of the analysis only because our focus was on the dynamics between D-, A-, and T-type tumors within a DI of 0.9-2.2. The DI ranges for the D-, A-, and T-type tumors presented have been shown to represent three statistically significant different tumor entities with respect to DI, S-phase, PI, and stemline scatter index (SSI) [48].

\section{Age and tumor progression}

In this report, we introduced increasing age of $\mathrm{BC}$ patients as a parameter to follow genomic instability reflected in DI and alterations among D-, A-, and T-type tumors. We included patients under 60 years of age from the original sample of 1,280 patients (Figure 1) and related them to four accumulative SSI intervals (Figure 2). This covers a period of two to three decades. We consider this to be a more homogeneous population than patients in the whole age interval up to 90 years of age. Fluoroscopic treatment of the chest against tuberculosis indicated that BC tumors developed within 15-20 years after dropping of the atomic bomb in Japan [49]. A summary of Figure 2 is shown in Table 1 for D-, A1-, A2-, T-, and A3-type tumors. To demonstrate changes

Age at Breast Cancer Diagnosis and DNA-Index $n=1,280$

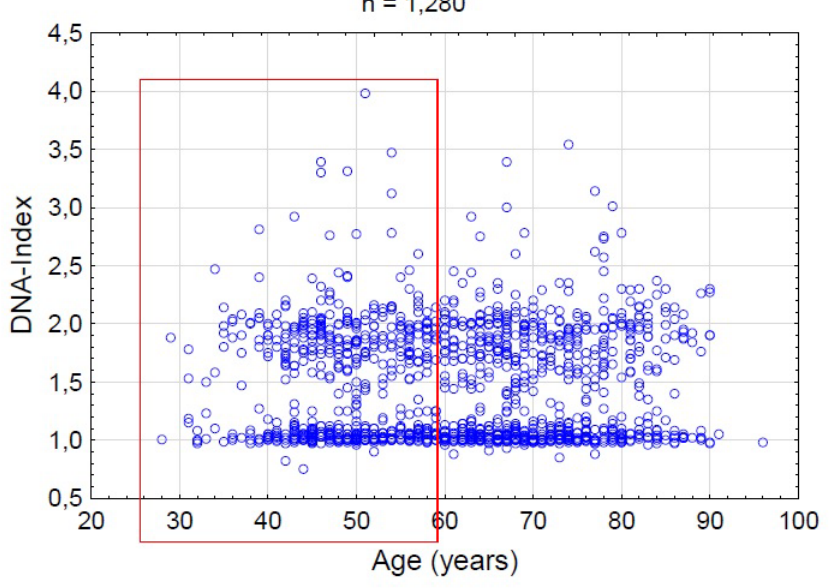

Figure 1: Distribution of DNA-indices in the whole population. The sample selection for analyzing the relationship between DNA-index and age of patients is shown within the red rectangle.

\begin{tabular}{|l|c|c|c|c|c|}
\hline Age $<\mathbf{6 0}$ years & $\begin{array}{c}\mathbf{0 , 9} \leq \mathrm{DI}<\mathbf{1 , 2} \\
\text { D-type }\end{array}$ & $\begin{array}{c}\mathbf{1 , 2} \leq \mathrm{DI}<\mathbf{1 , 4} \\
\text { A1-type }\end{array}$ & $\begin{array}{c}\mathbf{1 , 4} \leq \mathrm{DI} \mathbf{1 , \mathbf { 8 }} \\
\text { A2-type }\end{array}$ & $\begin{array}{c}\mathbf{1 , 8} \leq \mathrm{DI}<\mathbf{2 , 2} \\
\text { T-type }\end{array}$ & $\mathbf{D I} \geq \mathbf{2 , 2}$ \\
\hline $\mathrm{SSI}<5$ & $43 / 60$ & $2 / 60$ & $1 / 60$ & $11 / 60$ & $3 / 60$ \\
& $71,7 \%$ & $3,3 \%$ & $1,7 \%$ & $18,3 \%$ & $5 \%$ \\
\hline $\mathrm{SSI} \leq 8,8$ & $152 / 218$ & $8 / 218$ & $7 / 218$ & $40 / 218$ & $10 / 218$ \\
& $69,7 \%$ & $3,7 \%$ & $3,2 \%$ & $18,3 \%$ & $4,6 \%$ \\
\hline $\mathrm{SSI} \leq 15$ & $219 / 380$ & $14 / 380$ & $31 / 380$ & $101 / 380$ & $14 / 380$ \\
& $57,6 \%$ & $3,7 \%$ & $8,2 \%$ & $26,6 \%$ & $3,7 \%$ \\
\hline $\mathrm{SSI} \leq 30$ & $249 / 508$ & $18 / 508$ & $73 / 508$ & $146 / 508$ & $27 / 508$ \\
& $49 \%$ & $3,5 \%$ & $14,3 \%$ & $28,7 \%$ & $5,3 \%$ \\
\hline $\mathrm{SSI} \leq 50$ & $254 / 549$ & $18 / 549$ & $89 / 549$ & $165 / 549$ & $24 / 549$ \\
& $46,3 \%$ & $3,3 \%$ & $16,2 \%$ & $30,1 \%$ & $4,4 \%$ \\
\hline Comparison & $\mathrm{P}<0.001$ & & $\mathrm{P}<0.001$ & $\mathrm{P}<0.001$ & \\
\hline $\mathrm{SSI}<5 / \mathrm{SSI} \leq 50$ & & & & & \\
\hline
\end{tabular}

Table 1: Ploidy and SSI. Five ploidy entities and five increasing SSI levels of relative units compared among women $<60$ years of age. Numbers of D-type tumors decreased and T-, and A2-type tumors increased. All three changes were statistically significant. 
A

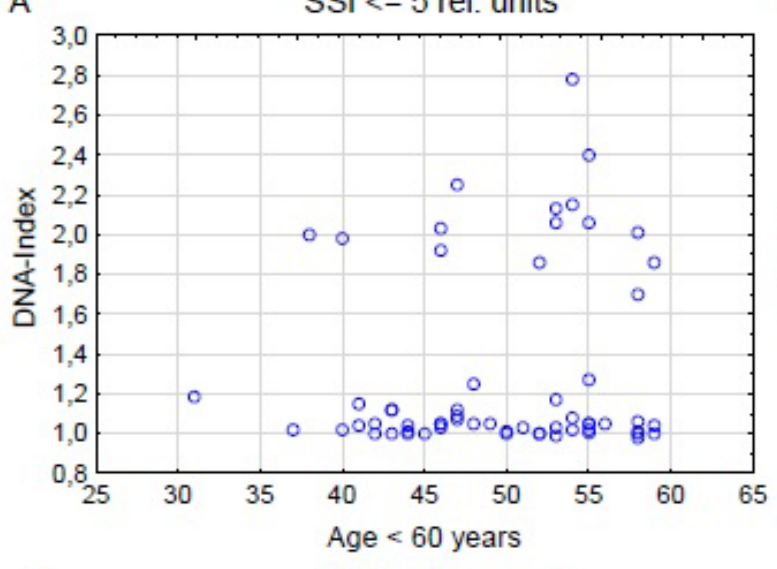

C

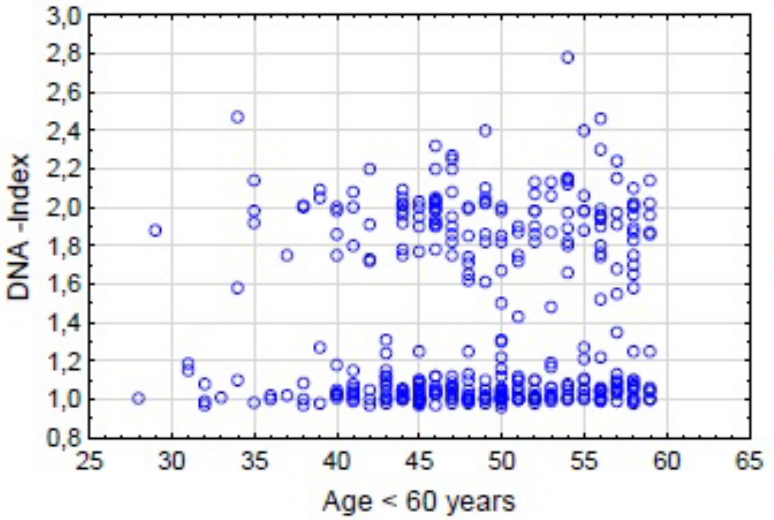

B

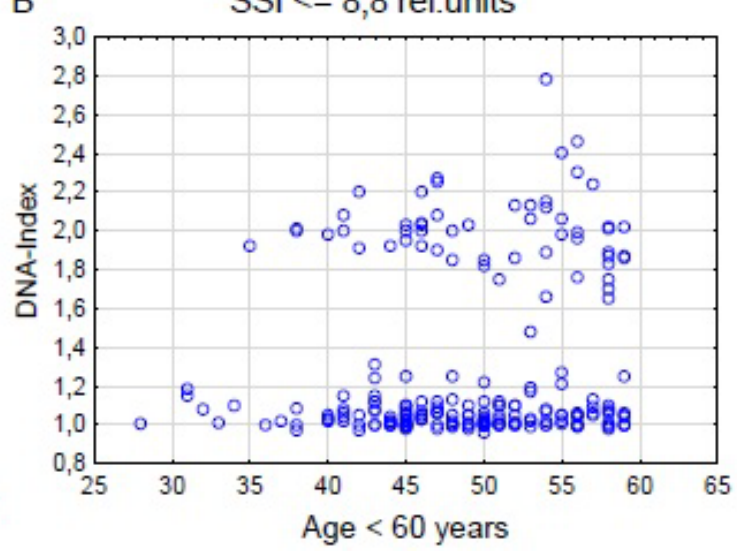

$\mathrm{D}$

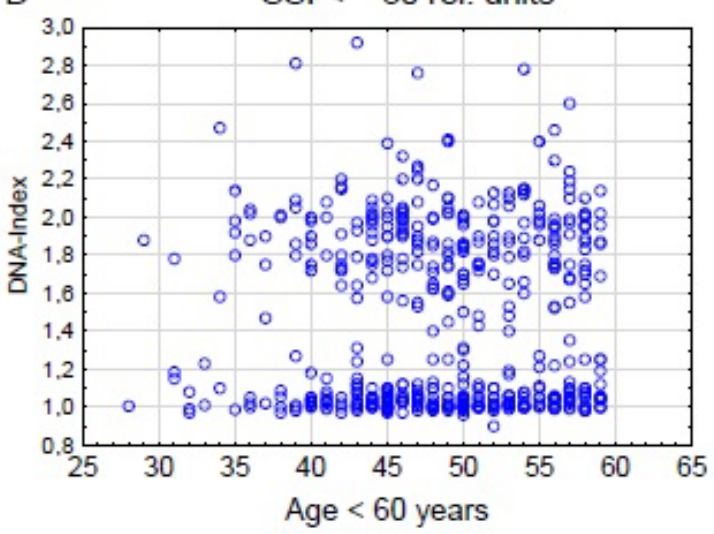

Figure 2: Genomic instability and patient age. The DNA index was plotted for patients aged $<60$ years in four increasing SSI intervals: $\leq 5$ relative units (A), $\leq 8.8$ relative units $(B), \leq 15$ relative units $(C)$, and $\leq 30$ relative units (D). D- and T-type tumors dominated the two first SSI intervals, after which the triploid DI interval $1.2-1.8$ increased, in particular the DI interval 1.4-1.8. Some few tumors with DNA-Index $>3.0$ were ecluded.

in the relationship between ploidy entities and SSI, two dimensional plots were drawn and we extended the four accumulative SSI intervals in Figure 2 to 35 short SSI intervals reflecting attained accumulated numbers of each ploidy entity per step and estimated the total numbers per interval. We calculated the ploidy percentage at each interval for those with significant change in Table 1: D-, A2 and T-type tumors. This enabled us to determine how the ploidy entities changed with respect to increases in SSI values. The accumulated curves revealed trends and smoothed out minor deviations, while giving stronger correlation than raw data.

\section{Feulgen staining}

This was carried out as previously described [48].

\section{Stemline-scatter-index (SSI)}

To create a large-scale simulation at a low level of resolution, we estimated genomic instability and proliferative activity using the coefficient of variation for the tumor stemline G1 peak (G1CV) and the S-phase fraction (SPF) for each patient, as well as the percentage of cells for each tumor with a DNA content above the G2 DNA level (exceeding the G2 rate, Exc-G2). Thus, the SSI includes G1CV+SPF+Exc-G2, all expressed as percentages. In previous reports, when first presenting the SSI parameter, we used an SSI cutoff value of $8.8 \%$ to differentiate between tumors representing significantly scattered DNA histograms $(\mathrm{SSI}>8.8 \%)$ and those with insignificantly scattered ones (SSI $\leq 8.8 \%)$ [50].We continued to apply this limit in the present study mainly for historical reasons. However, the progress of tumor growth should be considered as a continuum rather than an attainment of specific cutoff points. By relating the three ploidy entities to increasing SSI values and ages of the patients, the development of genomic heterogeneity and proliferative activity was followed. G1CV and SPF were found to increase before the appearance of Exc-G2 cells, so we applied an equation from a three-dimensional surface using xyz variables in which Exc-G2 is denoted as z: $\mathrm{z}=0.0152+0.0508 \mathrm{x}+0.0506 \mathrm{y}$. Thus, G1CV (x) and SPF (y) contribute equally to the combined SSI parameter. Previous studies using comparative genomic hybridization (CGH) showed an increasing number of gains, some losses and regional amplifications of chromosomes during breast tumor progression that will generate change in the G1CV [32].

\section{Lognormal distribution of parameters}

A variable might be modeled as lognormal if it can be thought of as the multiplicative product of many independent random variables, each of which is positive and none of which has a decisive influence. We investigated G1, S and G2 phases, DI values and G1CV parameters obtained from image analysis of Feulgen-stained tumor cells from 
every individual tumor specimen. These parameters were tested in a probability-probability plot, comparing empirical against theoretical cumulative values in a lognormal curve, because growing cell populations represent multiple events during the passage through the cell cycle.

\section{DNA index distribution in histograms}

We analyzed the distribution of DNA indices in histograms of four increasing values of SSI intervals: SSI $\leq 8.8 ; 8.8<$ SSI $\leq 12 ; 12<$ SSI $\leq 15$; and $15<$ SSI $\leq 40$. Guided by these SSI intervals, we conducted stepwise alterations in the histograms of D-, A-, and T-type tumors and analyzed the change in interrelation between the ploidy entities during increasing SSI relative values.

\section{Statistical analysis}

Statistical analysis was performed using the STATISTICA software package (StatSoft Inc., Tulsa, OK, USA). Statistical significance for categorical variables was calculated using the chi-squared test, and an independent $t$-test was used for continuous variables. Linear regression was performed for the correlation test. Statistical significance was assumed if $P<0.05$. Analysis of survival curves was performed using the Kaplan-Meier method. Differences between curves were estimated using the log-rank test. The study design was approved by the ethics committee at Karolinska Institutet, Stockholm, Sweden (2013/707$31 / 3)$.

\section{Results}

In Figure 1, we show the sample selected for the study of age against DI from the whole population of 1,280 breast cancer patients. The observation of two peaks of tumors in the DI region $\geq 2.2$ indicates two main populations under and over 60 years of age (Figure 1) (See Materials and Methods: Age and tumor progression) we examined ploidy change during increasing age from the youngest up to women $<60$ years $(n=588)$ through four extensions of accumulated SSI intervals from SSI $\leq 5$ to SSI $\leq 30$ relative units (Figure 2A-D). Along with increasing SI values, we found a significant decrease in D-type tumors and significant increases in T-, and A2-type tumors. The A1-, and A3type tumors remained stable at a level of $5 \%$ (Table 1).

Using increasing SSI against DI instead of age (Figure 3A) we present in Figure 3B a two-dimensional plot of the DI interval 1.4 $\leq \mathrm{DI}<2.2$ representing tumors from the hypertriploid (A2-type) to the hypertetraploid (A3-type) tumors. The curve shows at the $95 \%$ confidence interval a significant negative slope, demonstrating a transfer of T-type tumors to the hypotetra-, and reaching the A2-type DI region during increasing SSI (compare Figures 2C and 2D).

Among patient age $\leq 45$ years and $\mathrm{SSI} \leq 5$ relative units, there were only D-, (87\%) and T-type (13\%) tumors (Figure 2A). Keeping the SSI parameter at that low level and estimating the whole sample aged $<60$ years for dispersion of DNA entities, we found $73.3 \%$ D-type tumors, 16.7\% T-type tumors, 5\% A-type tumors and 5\% A3-type tumors. These data indicate that despite keeping the SSI parameter stable, genomic instability continues to increase by its own power once it has started.

\section{Looking for trends}

The total sample in Table 1 represented 549 BC patients that could be subdivided into 35 increasing SSI levels to create accumulating curves revealing trends in the tumors showing significant change in Table 1, i.e., D-, A2-, and T-type tumors. In Figure 4A, the D-type tumors showed a significantly negative slope in relation to increasing SSI

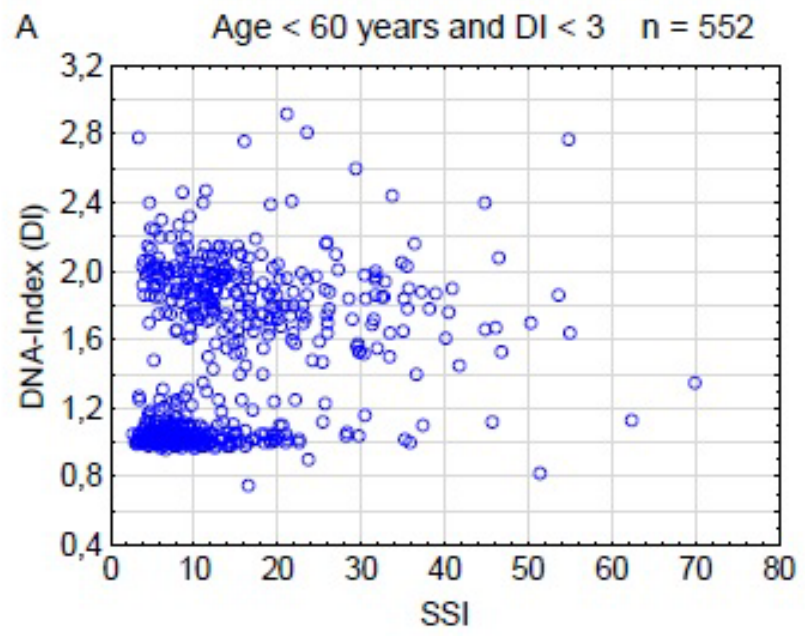

B $\quad r=-0.32 \quad P<=0.001 n=263$

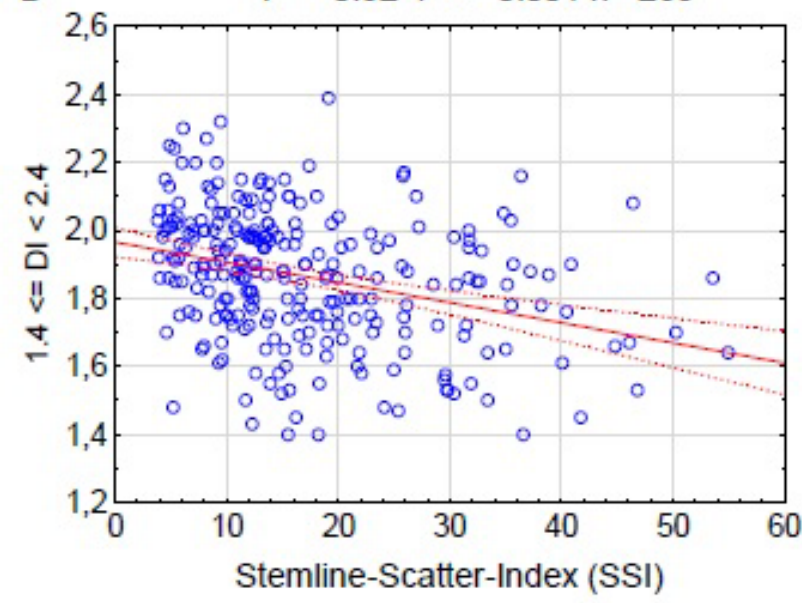

Figure 3: Patient age $<60$ years and genomic instability. (A) The DNA index plotted against SSI for patients aged $<60$ years shows that $D$ - and T-type tumors dominate at $\mathrm{SSI} \leq 8.8$ relative units $(88.5 \%)$. (B) Within the DI interval $1.4-2.2$ reflecting tetra-, hypotetra-, and hypertriploid tumors, a statistically significant negative slope was apparent.

relative units, while the T-type tumors showed a significantly positive slope as expected from Table 1 . From SSI $\approx 12$ to 15 relative units, there is a reduction in the D-type curve (Figure $4 \mathrm{~A}$ ) and a more prominent increase in the T-type curve (Figure 4B). Between the A2-type curve and SSI, there was a statistically significant positive correlation without deviation (Figure 4C). In Figure 4D, there was a strong increase of accumulating A2-type tumors among patients aged from 35 to 60 years. The A1-type tumors showed a much slower increase.

\section{Lognormal probability-probability cumulative population}

Our results showed that T-type tumors appear early with a low SSI value (Figure 2A) and later have an increased SSI value around 12-15 relative units (Figure 4B). To further analyze the growth dynamics, cell cycle parameters were analyzed in Feulgen-stained tumor specimens assessed for G1, G2 and SPF phases together with DI, G1CV and tumor size. A large variation in DNA distribution was observed among individual BC patients, with some peaking only in G1, and others having additional cells in SPF, and/or a G2 peak of variable 
A

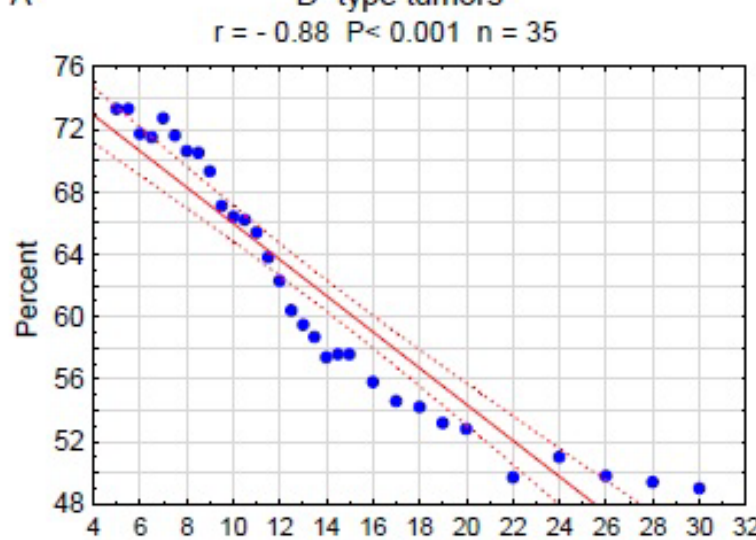

$\mathrm{C}$

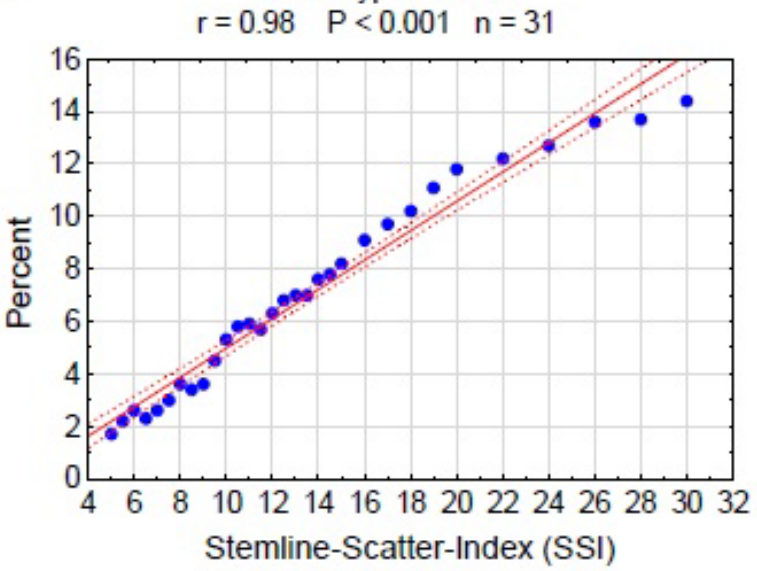

B
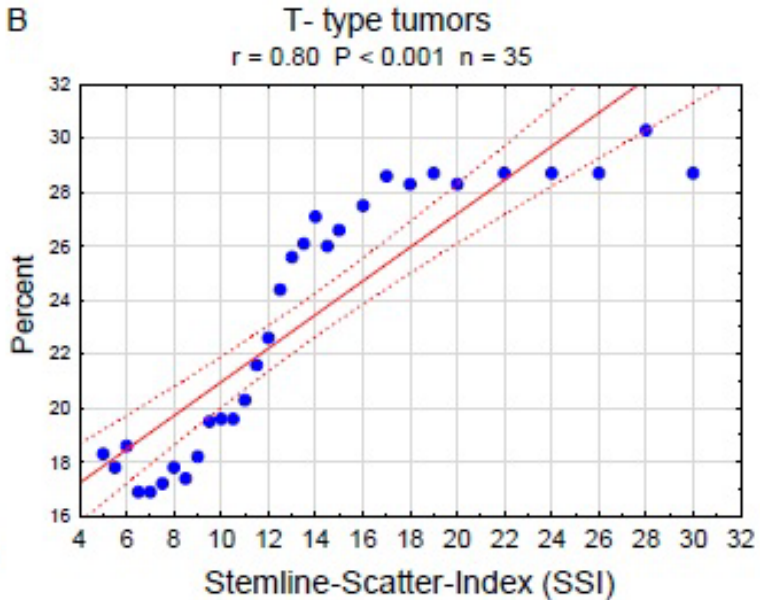

A1- and A2- type tumors

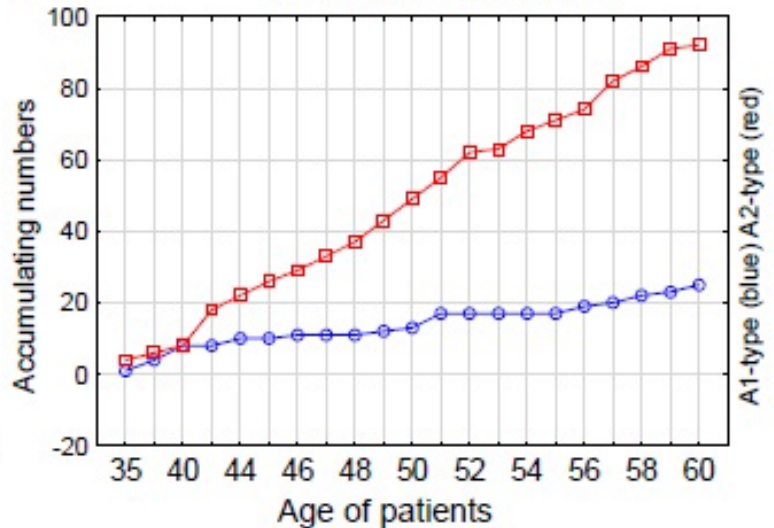

Figure 4: Line plot relationships between ploidy entities and SSI. Accumulated achieved values for D- (A), T-, (B) and A2-type tumors (C) are shown against increasing SSI relative units. The D-type tumor plot was significantly negative and the A2-, and T-type tumor plots significantly positive. The T-type tumors increased close to SSI relative units=12, when a reduction in D-type curve was observed. For accumulating numbers of A1-, and A2-type tumors relative to increasing age of $B C$ patients, the A2-type tumors had a much stronger positive slope compared with the A1-type tumors (D).

size. We defined these patient data as a growing cell population fixed individually at different stages of the cell cycle. The outcome was analyzed as a lognormal probability-probability distribution simulation, with the empirical cumulative curve as the y-axis and the theoretical cumulative curve as the $\mathrm{x}$-axis. G1 values showed an initial delay in adaptation to the theoretical curve and thereafter deviated to a steeper positive slope (Figure 5A), probably reflecting the $\mathrm{G} 0$ resting state at the beginning of the G1 phase. The SPF and G2 phase closely fitted the theoretical curves (Figures 5B and 5C), as did the G1CV and tumor size when included (not shown). This suggested that the cell population was derived from many cell cycle regulating parameters of which no individual had a decisive influence. DI values on the contrary deviated from the theoretical curve indicating an initial increase in DNA content in a fraction at the empirical cumulative interval of $0-0.2$, and a second increase at the empirical cumulative value of 0.6-0.8 (Figure 5D). The first appearance of T-type tumors can be seen early in Figure 2A at a low SSI value.

\section{DNA index histograms of increasing SSI intervals}

Because of the indication of a second increase in T-type tumors (Figure 5D), we next used four separated SSI intervals (SSI $\leq 8.8$,
8.8-12, 12-15, and 15-40 relative units) to create four histograms of DI values (Figure 6) including the whole sample of 1,280 patients. There was an increase in T-type shown from Figure $6 \mathrm{~A}$ to $6 \mathrm{~B}$ and a more prominent increase in T-type tumors in Figure $6 \mathrm{C}$ with a peak at DI=2.0-2.1. In the SSI interval 15-40, the peak moved to the hypotetraploid and hypertriploid positions in the DI range 1.7-1.8 (Figure 6D). A comparison with D-type tumors between Figures 6A and $6 \mathrm{C}$ showed a significant decrease from $67.6 \%(384 / 568)$ to $36.1 \%$ $(52 / 144)(P<0.001)$, while a significant increase was observed in T-type tumors between Figure $6 \mathrm{~A}$ from $21 \%(119 / 568)$ to $40.3 \%(58 / 144)$ in Figure 6C $(P<0.001)$. Tumors in the DI interval $1.6 \leq \mathrm{DI}<1.8$ increased significantly between Figure 6C $(11.1 \%)$ and Figure $6 \mathrm{D}(20.4 \% ; P<0.02)$. These findings suggest that a significant transition to a tetraploid position occurred in tumors (Figure 6C) and a subsequent transfer to the hypertriploid DI region (Figure 6D) (compare with Figure 3B). The mean tumor size $(\mathrm{mm})$ in the four SSI intervals in Figure 6 was (A) 19.0 \pm 13.3 , (B) $20.9 \pm 15.6$, (C) $17.7 \pm 8.5$ and (D) $21.1 \pm 12.2$. This showed that tetraploidization occurred in the size interval of $10-20 \mathrm{~mm}$.

\section{Second tetraploidization}

To visualize the evidence for a second tetraploidization (Figures 4B, 
A

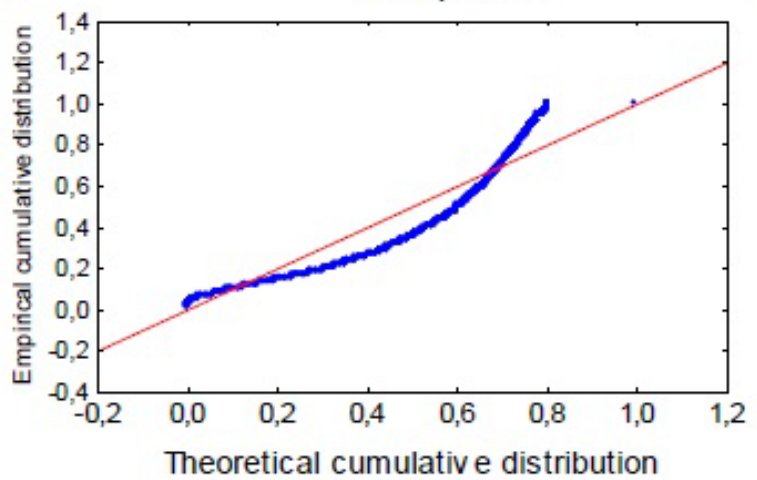

$\mathrm{C}$

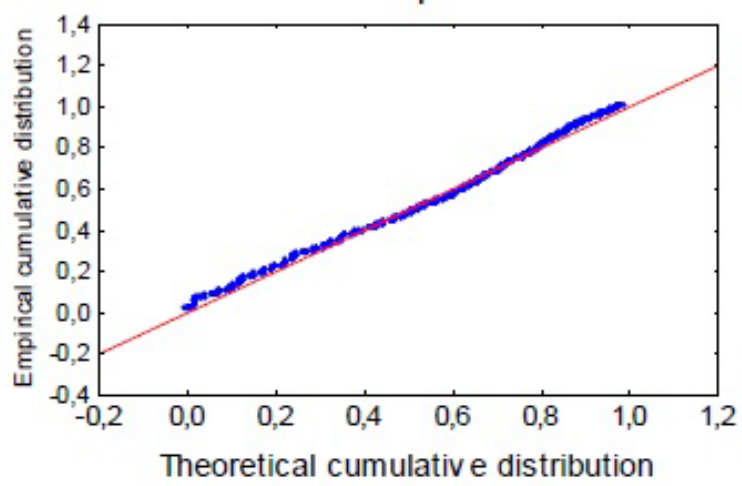

B

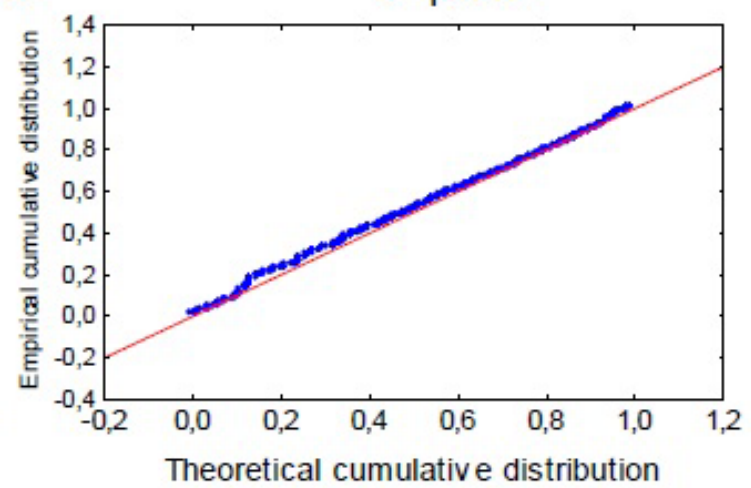

D

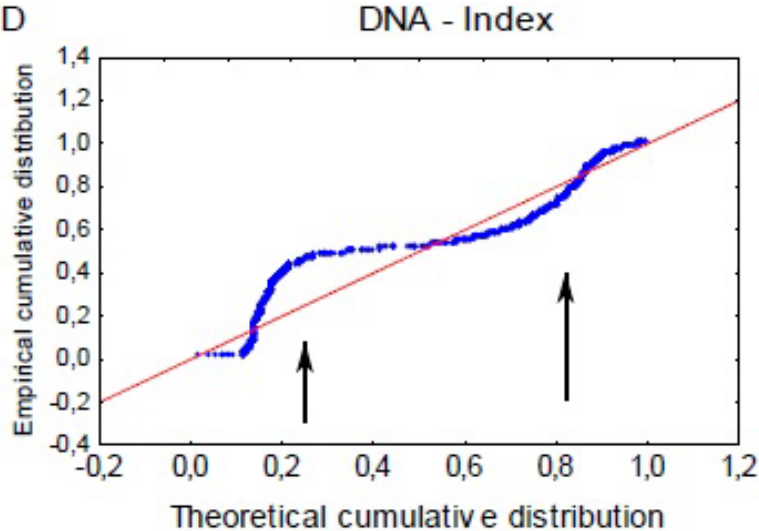

Figure 5: Probability-Probability plot. Empirical cumulatively estimated cell cycle parameters were plotted against a theoretical cumulative distribution. A deviation from the theoretical curve was found for the G1-phase curve (A) and was most prominent for the DNA index (D). At the two arrows, a prominent increase in the DI-curve was found. Values for SPF (B) and G2 phase (C) appear well adjusted to the theoretical curve.

$5 \mathrm{D}$ and $6 \mathrm{C}$ ), the curve in Figure 4B was divided into separate intervals by registering numbers of marks between two integers. D-, and T-type tumors were then counted and expressed as a percent of total tumors for each interval. The method increased the degree of resolution. The result shown in Figure 7 demonstrates a tetraploidization in the SSI interval from 12 to 15 relative units.

\section{Prerequisites for a hypoxic situation}

Tumors growing in size can reach a state of hypoxic threat. We observed a strong correlation between tumor size and auxiliary lymph node metastasis (ALNM). Analysis of all 1,280 patients showed that the mean size of tumors with ALNM $=0$ was significantly smaller, at $17.4 \pm$ $9.46 \mathrm{~mm}(\mathrm{n}=683)$, than that of tumors with ALNM $>0$, at $24.3 \pm 12.5$ $\mathrm{mm}(\mathrm{n}=418)(P<0.00001)$. Tumors with $\mathrm{ALNM}=0$, within the $10-20$ $\mathrm{mm}$ size interval $(\mathrm{n}=595)$ and $12<\mathrm{SSI} \leq 15$ have a mean size of $14.1 \pm$ $2.28 \mathrm{~mm}$ and therefore will be at a high risk of hypoxia, because breast tumors of that size were previously reported to have a level of VEGF [40] lower than that required for lymph vessels to penetrate into the tumor mass. We observed a mean tumor size of $17.5 \pm 8.5 \mathrm{~mm}$ in Figure 6C where a high level of T-type tumor was shown for SSI interval 12-15 relative units.

\section{Examination of two A-type populations}

Based on our findings that A-type tumors mainly derived from the loss of genetic material in unstable T-type tumors, which occurred twice during breast tumor progression (Figures 3B, 4B, 5D and $6 \mathrm{C}$ ), a bimodal survival time might be expected among A-type tumors. From the total sample of 1,280 patients, 253 had A-type tumors in the triploid DI region. Of these, up to the year 2010, 88 patients died from BC. As shown in Figure 8, two populations of these deceased patients could be identified based on survival time: the first surviving $\leq 80$ months, and the second 80-240 months. The mean survival time for the first group was significantly lower at 31.3 \pm 17.2 months $(n=62)$ than that of the second group at $131 \pm 36.4$ months $(n=26)(P<0.0001)$. Within the DI range of $1.2-1.65$ on the $\mathrm{y}$-axis, the two populations differed by a survival time of 40 months with no overlap in the interval (Figure 8A). A significant difference in tumor size was observed between the two different populations, with a significantly larger mean tumor size in the first group (27.2 $\pm 13.3 \mathrm{~mm})$ compared with the second $(16.0 \pm 7.4 \mathrm{~mm} ; P=0.0005)$. The Kaplan-Meier method was used to investigate tumors 10$20 \mathrm{~mm}$ in size $(\mathrm{n}=633)$, when tetraploidization occurred (Figure 6C), without selecting for SSI values, and revealed a bimodal slope for A-type tumors on each side of the 80-month, post-diagnosis survival period (Figure 8B). This indicates the presence of two subpopulations of A-type tumors. The log-rank test revealed that the difference between D- and A-type tumors in Figure $8 \mathrm{~B}$ was significant $(P<0.01)$. 
Citation: Sennerstam RB, Strömberg JO (2015) Genomic Instability or One-Gene Theory for Tumor Progression: A Breast Cancer Study. J Carcinogene Mutagene 6: 223. doi:10.4172/2157-2518.1000223

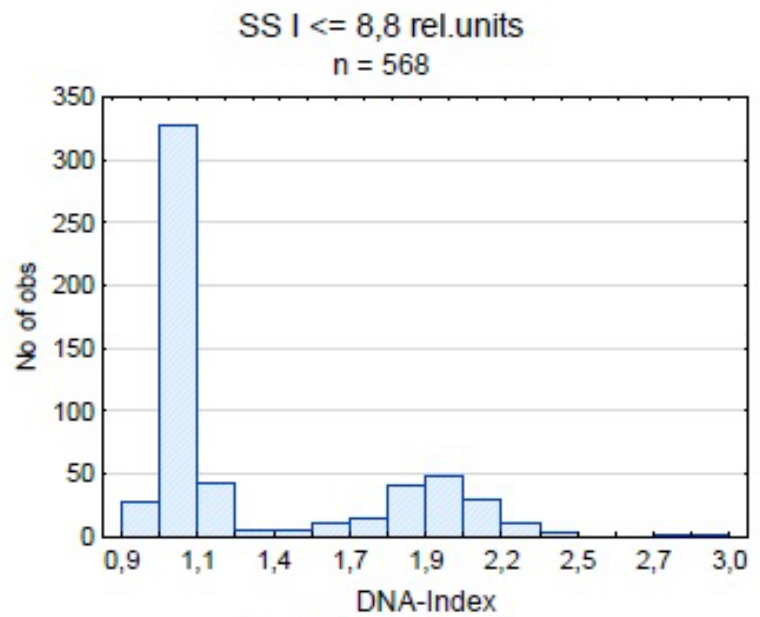

$12<\mathrm{SSI}<=15$ rel.units

$\mathrm{n}=144$

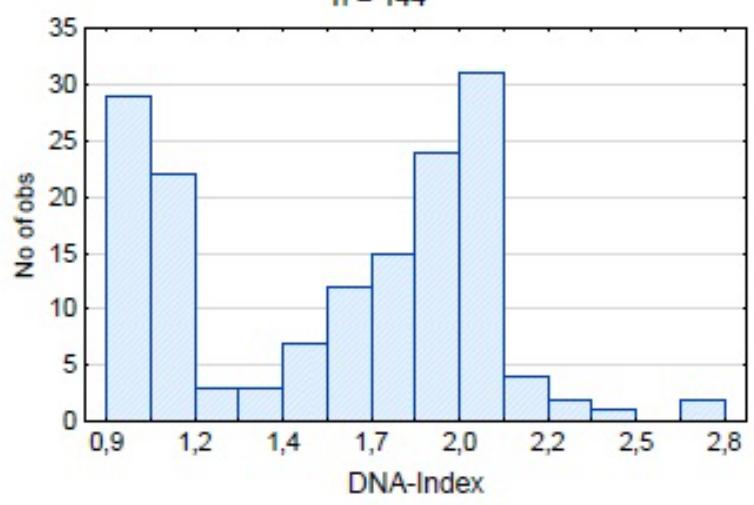

$8,8<\mathrm{SSI}<=12$

$n=215$

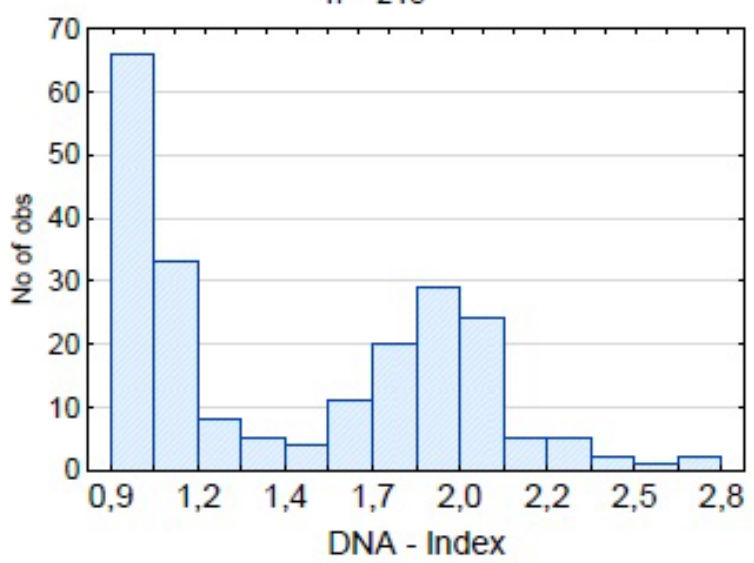

1 b $<\mathrm{SSI}<=4 \mathrm{U}$ rel.units

$\mathrm{n}=314$

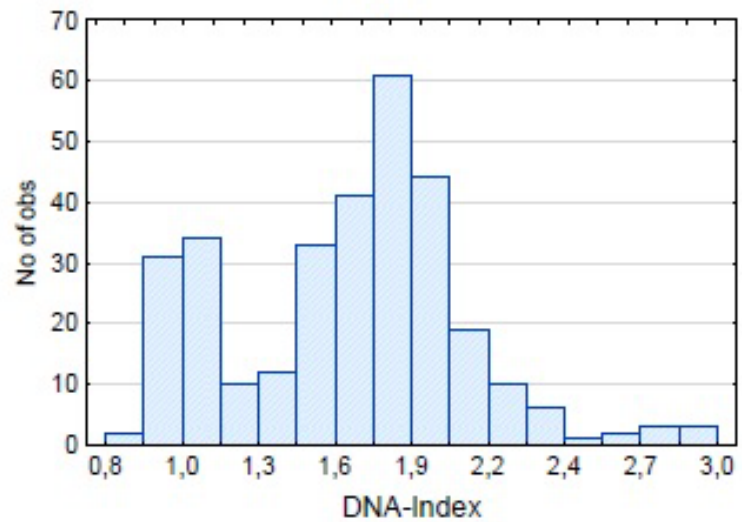

Figure 6: DNA histograms in increasing SSI intervals. (A) D-type tumors predominate for SSI $\leq 8.8$ relative units with some T- and A-type tumors. A slightly enhanced T-type population is seen in SSI interval 8.8-12 (B) and reaches a peak in the SSI interval 12-15 (C). A-type tumors dominate in the 15-40 SSI interval (D).

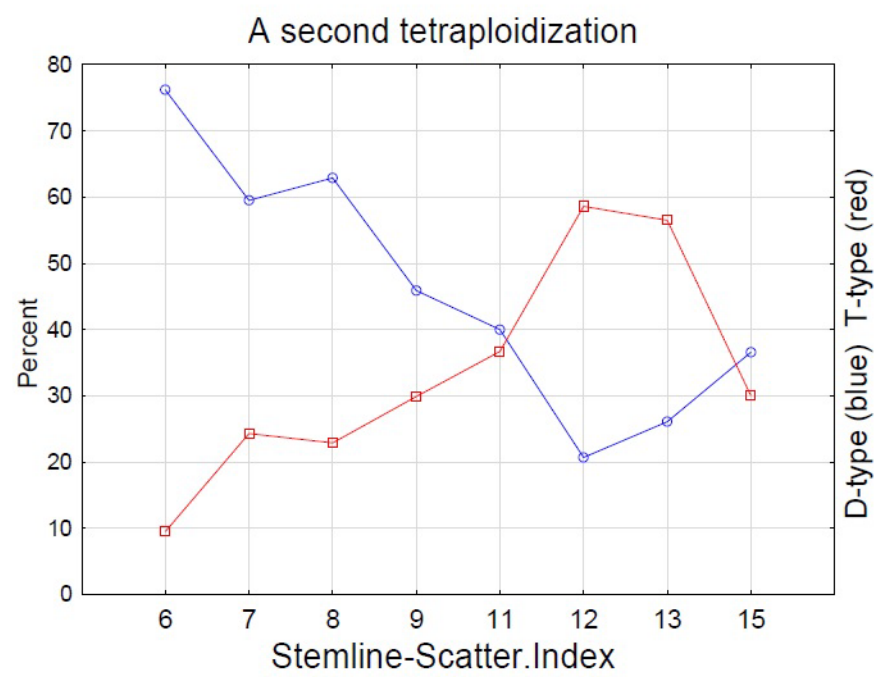

Figure 7: Second tetraploidization. Transforming the curves A) and B) in Figure 4 from accumulating plots to mean of intervals between two integers of SSI relative values achieved a higher level of resolution and a clear cut tetraploidization appeared. 
8 A A-type tumors and months to death

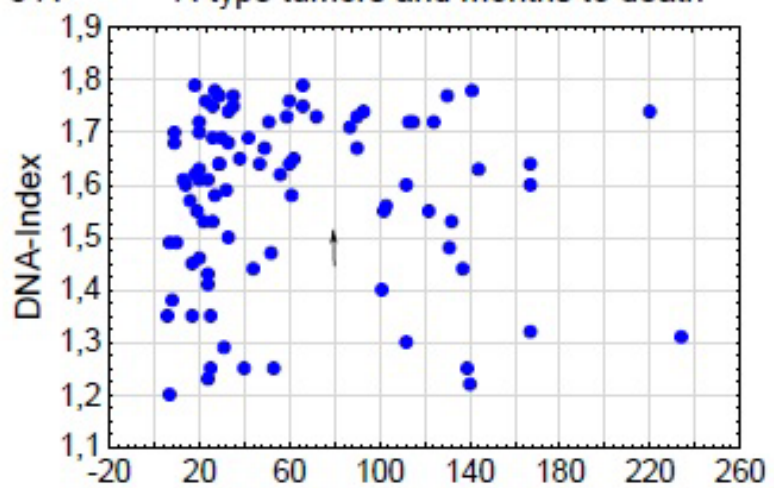

$8 \mathrm{~B}$

- Complete * Censored

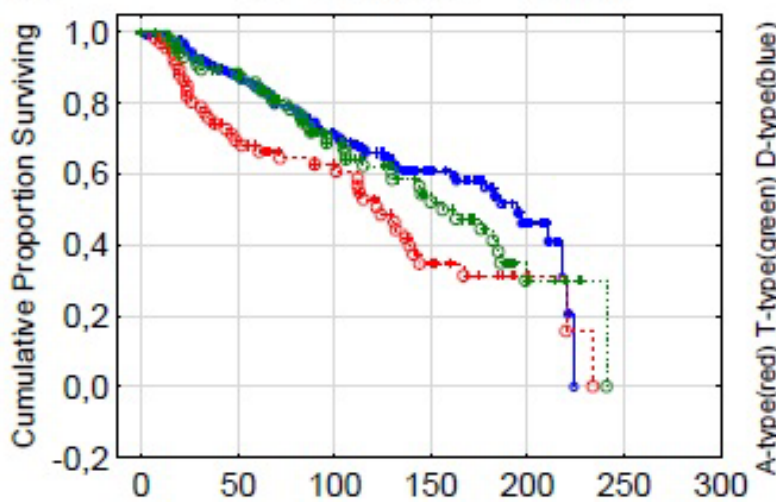

Figure 8: Patient survival with A-type tumors. (A) The plots shows the time (in months) until death for patients with A-type tumors in the triploid DI region $(1.2 \leq \mathrm{Dl}<1.8)$. Two populations of tumor appeared on both sides of the 80 -months survival point, which differed significantly in both survival time and size. Patients experiencing longer survival had tumors significantly smaller in size compared with short-term survivors. (B) Kaplan-Meier survival curves are shown for the three ploidy types (DA-T) within the tumor size interval of 10-20 $\mathrm{mm}$, within which the second tetraploidization occurred. A bimodal distribution of A-type tumors is apparent.

\section{Age and death in breast cancer}

The positive relationship between increasing age and genomic instability cannot be interpreted as death from BC increases with age. Other parameters contribute to the outcome. It is known that women under 40 years of age have a higher death rate than the elder women. In an 18-year follow up of our sample 25 of 49 women $<40$ years old died from BC (59\%) compared to 66 of 231 women (28.6\%) aged 50-60 years $(P<0.002)$. PI for women $<40$ years was $19.2 \pm 14.4 \%$ and for women aged $50-60$ years was $14.1 \pm 12.6 \%(P<0.02)$. Among women $<50$ years of age $(n=298) 87$ developed distant metastasis $(29.2 \%)$ in 18 years of follow up and among women $\geq 50$ years of age $(n=966) 172$ had distant metastasis $(17.8 \% ; P<0.001)$. Women aged $50-69$ years were included in mammography screening every second year, which increases survival rates [51]. Mean tumor size for women aged $<50$ years was $20.5 \pm 12.0$ $\mathrm{mm}(\mathrm{n}=294)$ and for screened women aged $50-69$ years $17.8 \pm 10.4$ $\mathrm{mm}(\mathrm{n}=574 ; \mathrm{P}<0.001)$, a size difference that favors survival among screened women.

\section{Discussion}

Tetraploidization is an evolutionary conserved phenomenon that can occur in humans in response to infections and overloaded or aged tissues. Tetraploidization is not per se carcinogenic and needs no specific gene mutation. However, tetraploidization in a tumor transformed diploid cell increases the risk for failures in the octoploid mitotic figures, causing destabilization within the genome transferred to the established tetraploid population, despite the absence of p53 mutation [24]. Normally the tetraploid state induces p53-dependent arrest of nontransformed mammalian cells in G1 phase [52]. Therefore oncogene mutations will enhance genomic instability. Keeping our rough parameter for genomic instability (SSI) at a stable low level, the generation of $\mathrm{T}$ and A-type tumors still increased during the time parameter represented by increasing age (Figure 2). We have shown that breast tumors in a hyperdiploid DI interval $(1.02<\mathrm{DI}<1.05)$ might trigger polyploidization [48]. In this report, we show that tetraploidization occurred early in tumor progression (Figures $2 \mathrm{~A}, 4 \mathrm{~B}$, $5 \mathrm{D}$ and $6 \mathrm{C}$ ). The genomic instability transferred the tumors mainly to the hypotetra-, and hypertriploid DI regions (Figures 3B and 4C and Table 1).

We suggest that the second tetraploidization (Figures 5D, 6C, and 7) to be caused by a stress factor due to lack of oxygen within growing tumors that reach a critical size around $10-20 \mathrm{~mm}$ and lack blood and lymph vessels for the supply of essential nutrition. Hypoxia has been shown to transform a whole cell population in the hyperdiploid BEX-c melanoma cell line after two 24-hour episodes of reduction in oxygen [53].

In clinical practice, we recommend the value of having DNA-ploidy data when characterizing the level of malignancy of human tumors. In view of the results in this report, this will provide information on how far the tumor has progressed in terms of genomic instability and malignancy potential even before mutation of oncogenes. If we look ahead a decade, drugs like the health food product resveratrol, which is derived from red grapes and has been found to inhibit tetraploidization [54], and might be a first line treatment for patients with diploid tumors as a block against tetraploidization in growing metastatic cells. In a further step, we can imagine a prophylactic treatment of reservatrol for high-risk people with inherited cancer genes.

\section{Conclusion}

This paper strengthens the theory of tetraploidization as being a metastable intermediate position driving, in its own right, tumor progression to a higher degree of genomic instability. We propose that tetraploidization occurs twice. In the first case it is a reaction to stress, and in the second, a state of oxygen deficiency, neither of which require mutation of oncogenes that regulate cell cycle checkpoints. However, mutations in an already unstable genomic environment will lead to increased instability by blocking tumor suppressor genes and apoptosis and will, therefore, provide many more clonal alternatives for the tumor to evolve and survive in the human body.

\section{Acknowledgements}

Clinical follow-up data were obtained from Oncology Center Stockholm, Sweden. Grants achieved from Karolinska Institutets Fund.

\section{References}

1. Otto SP (2007) The evolutionary consequences of polyploidy. Cell 131: 452462.

2. Otto SP, Whitton J (2000) Polyploid incidence and evolution. Annu Rev Genet 34: 401-437. 
3. Van de Peer Y, Maere S, Meyer A (2009) The evolutionary significance of ancient genome duplications. Nat Rev Genet 10: 725-732.

4. Heneen WK, Nichols WW, Levan A, Norrby E (1970) Polykaryocytosis and mitosis in a human cell line after treatment with measles virus. Hereditas 64 53-84.

5. Vliegen HW, Eulderink F, Bruschke AV, van der Laarse A, Cornelisse CJ (1995) Polyploidy of myocyte nuclei in pressure overloaded human hearts: a flow cytometric study in left and right ventricular myocardium. Am J Cardiovasc Pathol 5: 27-31.

6. Adler CP, Costabel U (1975) Cell number in human heart in atrophy, hypertrophy, and under the influence of cytostatics. Recent Adv Stud Cardiac Struct Metab 6: 343-355.

7. van der Heijden FL, James J (1975) Polyploidy in the human myometrium. Z Mikrosk Anat Forsch 89: 18-26.

8. Gentric G, Desdouets C, Celton-Morizur S (2012) Hepatocytes polyploidization and cell cycle control in liver physiopathology. Int J Hepatol 2012: 282430.

9. Lothschütz D, Jennewein M, Pahl S, Lausberg HF, Eichler A, et al. (2002) Polyploidization and centrosome hyperamplification in inflammatory bronchi. Inflamm Res 51: 416-422.

10. Hixon ML, Gualberto A (2003) Vascular smooth muscle polyploidization--from mitotic checkpoints to hypertension. Cell Cycle 2: 105-110.

11. Wagner M, Hampel B, Berhard D, Hala M, Zwerschke W, et al. (2001) Replicative senescence of human epithelial cells in vitro involves G1 arrest, polyploidization and senescence-associated apoptosis. Exp Gerontol 36: 1327 1347.

12. Walen KH (2006) Human diploid fibroblast cells in senescence; cycling through polyploidy to mitotic cells. In Vitro Cell Dev Biol Anim 42: 216-224.

13. Gallardo MH, Bickham JW, Honeycutt RL, Ojeda RA, Köhler N (1999) Discovery of tetraploidy in a mammal. Nature 401: 341

14. Storchova Z, Pellman D (2004) From polyploidy to aneuploidy, genome instability and cancer. Nat Rev Mol Cell Biol 5: 45-54

15. Ganem NJ, Storchova Z, Pellman D (2007) Tetraploidy, aneuploidy and cancer Curr Opin Genet Dev 17: 157-162

16. Galipeau PC, Cowan DS, Sanchez CA, Barrett MT, Emond MJ, et al. (1996) 17p (p53) allelic losses, 4N (G2/tetraploid) populations, and progression to aneuploidy in Barrett's esophagus. Proc Natl Acad Sci U S A 93: 7081-7084.

17. Shackney SE, Smith CA, Miller BW, Burholt DR, Murtha K, et al. (1989) Mode for the genetic evolution of human solid tumors. Cancer Res 49: 3344-3354.

18. Davoli T, de Lange T (2011) The causes and consequences of polyploidy in normal development and cancer. Annu Rev Cell Dev Biol 27: 585-610.

19. Duesberg P, Ruhong Li, Fabarius A, Hehlmann R (2006) Aneuploidy and cancer: From correlation to causation. In: Dittmar T, Zaenker KS, Smidt A (eds.) Infektions and Inflammation: Impact on Oncogenesis. Contrib Microbiol. Karger, Basel, pp. 16-44

20. Ganem NJ, Godinho SA, Pellman D (2009) A mechanism linking extra centrosomes to chromosomal instability. Nature 460: 278-282.

21. Lingle WL, Lutz WH, Ingle JN, Maihle NJ, Salisbury JL (1998) Centrosome hypertrophy in human breast tumors: implications for genomic stability and cell polarity. Proc Natl Acad Sci U S A 95: 2950-2955.

22. Lingle WL, Barrett SL, Negron VC, D'Assoro AB, Boeneman K, et al. (2002) Centrosome amplification drives chromosomal instability in breast tumo development. Proc Natl Acad Sci U S A 99: 1978-1983.

23. Fujiwara T, Bandi M, Nitta M, Ivanova EV, Bronson TR, et al. (2005) Cytogenesis failure generating tetraploids promotes tumorigenesis in P53-null cells. Nature 437: 1043-1047.

24. Auer GU, Heselmeyer KM, Steinbeck RG, Munck-Wikland E, Zetterberg AD (1994) The relationship between aneuploidy and p53 overexpression during genesis of colorectal adenocarcinoma. Virchows Arch 424: 343-347.

25. Duesberg P, Li R (2003) Multistep carcinogenesis: a chain reaction of aneuploidizations. Cell Cycle 2: 202-210.

26. Duesberg P, Stindl R, Hehlmann R (2000) Explaining the high mutation rates of cancer cells to drug and multidrug resistance by chromosome reassortments that are catalyzed by aneuploidy. Proc Natl Acad Sci U S A 97: 14295-14300.
27. Duessberg P (2005) Does aneuploidy or mutation start cancer? Science 307 41.

28. Nowell PC (1976) The clonal evolution of tumor cell populations. Science 194 23-28

29. Weaver BA, Cleveland DW (2006) Does aneuploidy cause cancer? Curr Opin Cell Biol 18: 658-667.

30. Storchova Z, Pellman D (2004) From polyploidy to aneuploidy, genome instability and cancer. Nat Rev Mol Cell Biol 5: 45-54

31. Heng HH, Bremer SW, Stevens JB, Horne SD, Liu G, et al. (2013) Chromosoma instability $(\mathrm{CIN})$ : what it is and why it is crucial to cancer evolution. Cancer Metastasis Rev 32: 325-340.

32. Blegen H, Will JS, Ghadimi BM, Nash HP, Zetterberg A, et al. (2003) DNA amplifications and aneuploidy, high proliferative activity and impaired cell cycle control characterize breast carcinomas with poor prognosis. Anal Cell Patho 25: 103-114.

33. Fabarius A, Hehlmann R, Duesberg PH (2003) Instability of chromosome structure in cancer cells increases exponentially with degrees of aneuploidy. Cancer Genet Cytogenet 143: 59-72.

34. Duesberg P, Stindl R, Hehlmann R (2000) Explaining the high mutation rates of cancer cells to drug and multidrug resistance by chromosome reassortments that are catalyzed by aneuploidy. Proc Natl Acad Sci U S A 97: 14295-14300.

35. Luoto KR, Kumareswaran R, Bristow RG (2013) Tumor hypoxia as a driving force in genetic instability. Genome Integr 4: 5 .

36. Huang LE, Bindra RS, Glazer PM, Harris AL (2007) Hypoxia-induced genetic instability--a calculated mechanism underlying tumor progression. J Mol Med (Berl) 85: 139-148

37. Vaupel P, Harrison L (2004) Tumor hypoxia: causative factors, compensatory mechanisms, and cellular response. Oncologist 9 Suppl 5: 4-9.

38. Dales JP Garcia S, Meunier-Carpentier S, Andrac-Meyer L, Haddad O, et al. (2005) Overexpression of hypoxia-inducible factor HIF-alpha predicts early relapse in breast cancer: retrospective study in a series of 745 patients. Int $J$ Cancer 116: 734-739.

39. Brown NS, Bicknell R (2001) Hypoxia and oxidative stress in breast cancer. Oxidative stress: its effects on the growth, metastatic potential and response to therapy of breast cancer. Breast Cancer Res 3: 323-327

40. Toi M, Inada K, Hoshina S, Suzuki H, Kondo S, et al. (1995) Vascular endothelia growth factor and platelet-derived endothelial cell growth factor are frequently coexpressed in highly vascularized human breast cancer. Clin Cancer Res 1 : 961-964

41. Caspersson TO (1979) Quantitative tumor cytochemistry--G.H.A. Clowes Memorial Lecture. Cancer Res 39: 2341-2345.

42. Auer GU, Caspersson TO, Wallgren AS (1980) DNA content and survival in mammary carcinoma. Anal Quant Cytol 2: 161-165.

43. Fallenius AG, Auer GU, Carstensen JM (1988) Prognostic significance of DNA measurements in 409 consecutive breast cancer patients. Cancer 62: 331-341.

44. Shapiro HM (1989) Flow cytometry of DNA content and other indicators of proliferative activity. Arch Pathol Lab Med 113: 591-597.

45. Dressler LG, Bartow SA (1989) DNA flow cytometry in solid tumors: practica aspects and clinical applications. Semin Diagn Pathol 6: 55-82.

46. Duesberg P, Rausch C, Rasnick D, Hehlmann R (1998) Genetic instability of cancer cells is proportional to their degree of aneuploidy. Proc Natl Acad Sci U S A 95: 13692-13697.

47. Ross JS, Linette GP, Stec J, Ross MS, Anwar S, et al. (2003) DNA ploidy and cell cycle analysis in breast cancer. Ann J Clin Pathol 120: S72-S84.

48. Sennerstam R, Strömberg J-O (2013) Hyperdiploidy tetraploidization and genomic instability in breast cancer - A case report study. J Carcinogen Mutagenesis 4: 2.

49. Boice JD Jr, Monson RR (1977) Breast cancer in women after repeated fluoroscopic examinations of the chest. J Natl Cancer Inst 59: 823-832.

50. Kronenwett U, Huwendiek S, Ostring C, Portwood N, Roblick UJ, et al. (2004) Improved grading of breast adenocarcinomas based on genomic instability. Cancer Res 64: 904-909. 
Citation: Sennerstam RB, Strömberg JO (2015) Genomic Instability or One-Gene Theory for Tumor Progression: ABreast Cancer Study. J Carcinogene Mutagene 6: 223. doi:10.4172/2157-2518.1000223

Page 10 of 10

51. Sennerstam R (2013) During introduction of mammography screening analysis of three tumor size intervals in screened and post-screened periods clarified the short and long term efficacy of screening. J Cancer Sci Ther s7: 006.

52. Andreassen PR, Lohez OD, Lacroix FB, Margolis RL (2001) Tetraploid state induces p53-dependent arrest of nontransformed mammalian cells in G1. Mol Biol Cell 12: 1315-1328.
53. Rofstad EK, Johnsen NM, Lyng H (1996) Hypoxia-induced tetraploidisation of a diploid human melanoma cell line in vitro. Br J Cancer Suppl 27: S136-139.

54. Lissa D, Senovilla L, Rello-Varona S, Vitale I, Michaud M, et al. (2014) Resveratro and aspirin eliminate tetraploid cells for anticancer chemoprevention. Proc Nat Acad Sci USA 111: 3020-3025. 